\title{
Stochastic Model in the Kardar-Parisi-Zhang Universality With Minimal Finite Size Effects
}

\author{
S.V. Ghaisas \\ Department of Electronic Science, University of Pune, \\ Pune 411007,India
}

September 6, 2018

\begin{abstract}
We introduce a solid on solid lattice model for growth with conditional evaporation. A measure of finite size effects is obtained by observing the time invariance of distribution of local height fluctuations. The model parameters are chosen so that the change in the distribution in time is minimum. On a one dimensional substrate the results obtained from the model for the roughness exponent $\alpha$ from three different methods are same as predicted for the Kardar-Parisi-Zhang (KPZ) equation. One of the unique feature of the model is that the $\alpha$ as obtained from the structure factor $S(k, t)$ for the one dimensional substrate growth exactly matches with the predicted value of 0.5 within statistical errors. The model can be defined in any dimensions. We have obtained results for this model on a 2 and 3 dimensional substrates. pacs60., 68.55-a,82.20.Fd
\end{abstract}


The KPZ equation [1] is one of the most studied stochastic equation in the field of growth.

$$
\frac{\partial h}{\partial t}=\nu_{0} \nabla^{2} h+\lambda(\nabla h)^{2}+\eta
$$

Here, $h(r, t)$ is the height function, $\lambda$ is coupling parameter, and $\eta(r, t)$ is Gaussian noise with the correlation $<\eta(r, t) \eta\left(r^{\prime}, t^{\prime}\right)>=\delta\left(r-r^{\prime}, t-t^{\prime}\right)$. First term is a linear term [2] referred as Edward-Wilkinson (EW) term. Applications and uses of KPZ equation have been well demonstrated [3]. In particular its use in understanding growth phenomenon has lead to vigorous activities in development of theoretical methods [4, 5], lattice models $[6,7,8]$ and numerical methods [9] built around Eq. (1). The critical exponents in $(1+1)$ dimensions are exactly obtainable [10]. However, in all the higher dimensions the determination of these exponents has been a difficult task. It is well known that this equation shows phase transition in dimensions higher than its critical dimension $(2+1)$ [10] as a function of its coupling parameter. For weak coupling case the coupling constant renormalizes to zero leading to a linear equation. For strong coupling case, the perturbation approach fails and other methods are required. However, obtaining exact values for the exponents has never been possible although the ranges in which exact values are expected to fall are evident from the available references $[4,5,6,7,8,9]$.

Various lattice models have been devised $[6,7,8]$ that are known to belong to the KPZ universality in the asymptotic region. Most of the models suffer from finite size effects arising from cutoff length $a$ and the substrate size $L$. One of the tests to probe the presence of finite size effects is to determine the growth exponents by different methods such as height-height (h-h) correlations, structure factor $S(k, t)=<h(k, t) h(-k, t)>$, from saturated widths $W_{\text {sat }}$, etc.. For any given lattice model, the values obtainable from these methods can be statistically different. Since all these models are expected to converge asymptotically to KPZ behavior, the apparent mismatch of the exponent values from different methods will be due to the finite size effects. On the other hand if a model gives same exponents within statistical errors, it is expected to be free of finite size effects. In the following we propose a model that we believe to belong to the KPZ universality and, in $(1+1)$ dimensions, it provides the values of the exponent $\alpha$ same within the statistical error, using three different methods of determination. This value also compares well with the exactly known value in $(1+1)$ dimensions, 0.5 .

We describe the model below and the changes therein for $(2+1)$ and $(3+1)$ 
dimensions. A site is chosen randomly and height at the site is increased by unity signaling random deposition on the substrate. The deposited atom is conditionally accomodated, otherwise evaporated. In $(1+1)$ dimensions the deposited atom is accommodated if both its neighbors have at least same height as the deposited one. Otherwise, largest of the height differences at the site $i$ and the nearest neighboring sites $, s_{d}=\max \left(h_{i}-h_{j}\right), j=i+1, i-1$, is obtained and accommodation is allowed according to the probability factor $e^{-s_{d}^{2} /\left(2 \sigma^{2}\right)}$. Thus $s_{d}$ is the largest local step. Choice of $\sigma$ depends upon the behavior of the model for the given value of $\sigma$. We choose the value of $\sigma$ that leads to minimum variation in the local height fluctuations. Model with such a $\sigma$ is expected to be least affected by the finite size effects [11]. It has been shown in reference [11] that a measure of finite size effects for a given lattice model can be obtained from the distribution of local height fluctuations. In this method, we define the local height $\left(h_{i}(t)\right)_{\text {local }}=h_{i}(t)-$ $\left(h_{i-1}(t)+h_{i+1}(t)\right) / 2$ with respect to the local reference as the average of nearest neighbor heights. Similarly we measure $\left(h_{i}(t+\Delta t)\right)_{\text {local }}$ where $\Delta t>$ $w(t) . w(t)$ is the width of the interface at $t$, and the inequality ensures that the difference between local heights measured at $t$ and $t+\Delta t$ are uncorrelated. Thus we measure the distribution of uncorrelated fluctuations $\Delta h(t)_{\text {local }}$ from the difference $\Delta h(t)_{\text {local }}=\left(h_{i}(t)\right)_{\text {local }}-\left(h_{i}(t+\Delta t)\right)_{\text {local }}$. Fig. 1 shows such a distribution for $\sigma=1.7$. Another distribution is obtained at later time and compared with the earlier one. In our case we have obtained distributions at $t=500 \mathrm{MLs}$ and $t=5000 \mathrm{MLs}$ for comparison with $\Delta t=100 \mathrm{MLs}$. Since the counts at $\Delta h(t)_{\text {local }}=0$ are largest in the distribution, the statistical error is minimum for zero fluctuation. We therefore use the parameter $P_{0}=$ $100\left(\frac{I_{500}-I_{5000}}{I_{500}}\right)$, where $I_{t}$ is the count at $\Delta h(t)_{\text {local }}=0$ to measure the time invariance of the distribution of $\Delta h(t)_{\text {local }}$ in $(1+1)$ and $(2+1)$ dimensions. In $(3+1)$ dimensions, $\Delta t$ and times for comparison are smaller due to the large computation times involved. Ideally $P_{0}$ should be zero. In the present context we look for a minimum value of $P_{0}$ as a function of $\sigma$. The ratios $P_{0}$ are obtained by averaging over large enough runs so that values of $P_{0}$ are statistically discriminated for different values of $\sigma$. It has been shown in reference [11] that this method is useful in identifying presence of finite size effects for any lattice model belonging to KPZ or EW universality. Fig. 2 shows the variation of $P_{0}$ with $\sigma$ for the model described. We have measured $P_{0}$ for the model with different $\sigma$ values on a substrate of length $L=40000$. As can be seen, the minimum occurs at $\sigma=1.7$. We have therefore used this 
value in $(1+1)$ dimensions. For other values of $\sigma$ we found that value of $\alpha$ as obtained from structure factor deviates from 0.5 and the linear range is also reduced on the log-log plot. This confirms the effectiveness of the method for determining finite size effects [11].

In $(2+1)$ dimensions the deposited atom is accommodated if three or more nearest neighbors have at least same height as its own. If this condition is relaxed to less number of in-plane neighbors, cross over due to EW region is obtained.The cross over is negligible when direct accommodation with three or four in-plane neighbors is allowed. For depositions at the site with less than three in-plane neighbors, the accommodation is decided from the largest of the four steps around the site using the exponential probability factor $e^{-s_{d}^{2} /\left(2 \sigma^{2}\right)}$. In $(2+1)$ dimensions we have observed that $\sigma=2.5$ shows minimum $P_{0}=0.002 \pm 0.0015 \%$. The substrate size is $L=400$.

In $(3+1)$ dimensions the deposited atom is accommodated if 5 or 6 nearest neighbors have at least same height as the deposited atom. We have used $\sigma=$ 4.5 in the simulations since this value gives minimum $P_{0}=0.0012 \pm 0.001 \%$. The $\Delta t=20 M L s$ and the distributions are compared for the times 50 MLs and 500 MLs for the substrate size of $L=100$.

We present results obtained from (h-h) correlations, $W_{\text {sat }}$ as a function of $L$, and structure factor. The (h-h) correlation is

$$
\begin{aligned}
G(x, t) & =\frac{1}{N} \sum_{x^{\prime}}\left(h\left(x+x^{\prime}, t\right)-h\left(x^{\prime}, t\right)\right)^{2} \\
& =x^{2 \alpha} f\left(\frac{x}{\xi(t)}\right)
\end{aligned}
$$

where, correlation length $\xi(t) \sim t^{1 / z}$. In the limit $x \rightarrow 0, f \rightarrow 1$. the width over a substrate of length $L$ as,

$$
\begin{aligned}
w_{2}(L, t) & =\frac{1}{N} \sum_{x}(h(x, t)-\bar{h}(t))^{2} \\
& =L^{2 \alpha} g\left(\frac{L}{\xi(t)}\right)
\end{aligned}
$$

Here $\bar{h}(t)$ is the average height at time $t$. It can be shown that [10] for times $t>>L, g\left(\frac{L}{\xi(t)}\right) \rightarrow$ const. , thus $w_{\text {sat }} \propto L^{2 \alpha}$. For $t<<L w_{2}(t) \propto t^{2 \beta}$. 
The structure factor $S(\mathbf{k}, t)$ is measured as

$$
S(\mathbf{k}, t)=<h(\mathbf{k}, t) h(-\mathbf{k}, t)>
$$

where $h(\mathbf{k}, t)=(1 / N) \sum_{\mathbf{x}}(h(\mathbf{x}, t)-\bar{h}(\mathbf{x}, t)) e^{i \mathbf{k} \cdot \mathbf{x}}$.

Figures (3), (4) and (5) summarize the results obtained for the model in $(1+1)$ dimensions. Fig. 3 shows the $\log -\log$ plot of $G(x, t)$ Vs. $x$. The straight line is fitted between $x=8$ and 500. The slope gives $\alpha=0.504 \pm 0.002$. Fig. 4 shows plot of $W_{\text {sat }}$ Vs. $L$ for $(1+1)$ dimensions. The least square fit to the points gives $\alpha=0.502 \pm 0.005$.

Fig. 5 shows $\log$-log plot of $\langle h(k, t) h(-k, t)>$ Vs. $k$. The straight line fit is between $k=0.03$ to 1.25 . The slope near $k=\pi$ tends to zero [12]. The slope is $2.003 \pm 0.021$. This gives [10] $\alpha=0.500 \pm 0.021$. Earlier, in reference [13] for the etching model, a slope $1.92 \pm 0.02$ was obtained in the range of $k=0.05$ to 0.1 , resulting in $\alpha=0.46$. This was considered to be one of the best value obtained for the existing lattice models by this method. Clearly the present model provides a better value. In the same reference, $\alpha=0.496$ is obtained from the $W_{\text {sat }}$. The apparent difference in the two $\alpha$ values indicates the presence of finite size effect for the etching model. In the present model, the slope is unaffected at smaller $k$ values. We have tested it up to $k=0.005$.

Above results show that the proposed model has minimal finite size effects. It further confirms the method introduced in reference [11] for determination of finite size effects in a lattice model. We have applied this method in $(2+1)$ dimensions and in $(3+1)$ dimensions to choose the model parameters corresponding to the minimal finite size effects.

In Fig. 6, we display the results of $W_{\text {sat }}$ Vs. $L$ in $(2+1)$ dimensions. The filled squares are the values calculated from the simulation results. The corresponding fit gives $\alpha=0.357 \pm 0.005$. Fig. 7 shows the log-log plot of $G(x, t)$ Vs. $x$. From its slope, $\alpha=0.355 \pm 0.001$. The line is fitted between $x=2$ to 50 . Thus both the methods give results matching within statistical margin.

In Fig. 6, we have also plotted the results of $W_{\text {sat }}$ Vs. $L$ for the model in $(3+1)$ dimensions. It gives $\alpha=0.289 \pm 0.005$. The $\alpha$ values obtained from these models in $(2+1)$ and $(3+1)$ dimensions are very close to those predicted in reference [14]. In this reference $\alpha=0.35702$ for $(2+1)$ dimensions and 0.28125 for $(3+1)$ dimensions.

We have also measured the $\beta$ values for these models from the log-log plots of $w_{2}(t)$ Vs. $t$. We obtain $\beta=0.332 \pm 0.001,0.221 \pm 0.002$ and 
$0.168 \pm 0.003$ for $(1+1),(2+1)$ and $(3+1)$ dimensions respectively . These values are consistent with the universal relation $\alpha+z=2$ for the KPZ equation [10].

In conclusion, we have developed a lattice model belonging to the KPZ universality with minimum finite size effects. That the finite size effects are minimum is evident from the results obtained. The $\alpha$ value using $W_{\text {sat }}$ Vs. $L, G(x, t)$ Vs. $x$, and from $S(k, t)$ Vs. $k$ plots are equal within statistical margin in $(1+1)$ dimensions. The simulation values are very close to the exact $\alpha, 0.5$. Measurement involving local height fluctuations is successfully used in determining the finite size effects in lattice models . The models with minimum finite size effects are expected to lead to the better accuracy in determining the exact exponents for KPZ growth in higher dimensions. In $(2+1)$ dimensions, we have obtained values close to $\alpha=0.36$ while in $(3+1)$ dimensions it is around 0.29 . Both these values are close to the earlier prediction in reference [14].

\section{References}

[1] M. Kardar, G. Parisi and, Y.C. Zhang, Phys. Rev. Lett. 56 , 889 (1986).

[2] S.F. Edwards and D.R.Wilkinson, Proc. R. Soc. London A381 ,17 (1982).

[3] F.D.Santos and M.M. Telo deGama, Review article to appear in Research Trends in Statistical Mechanics; also Cond-Mat /0409239.

[4] M. Lassig , Phys. Rev. Lett. 80, 2366 (1998).

[5] C. Castellano, M. Mrsili, M.A. Munoz, and L. Pietronero, Phys. Rev. E 59, 6460 (1999).

[6] J. M. Kim and J. M. Kosterlitz, Phys. Rev. Lett. 62 , 2289 (1989);T. Ala-Nissila, T. Hjelt, J.M. Kosterlitz and O. Venoloinen, J. Stat. Phys. 72, 207 (1993).

[7] D. E. Wolf and J. Kertesz, Europhys. Lett. 4, 651 (1987).

[8] P.Meakin, P. Ramanlal, L.M. Sander, and R.C. Ball, Phys. Rev A 34, 5091 (1986); F. Family and T. Vicsek, J. Phys. A 18, L75 (1985). 
[9] L. Giada, A. Giacometti, and M. Rossi, Phys. Rev. E 65, 036134 (2002).

[10] A.L. Barabasi and H.E. Stanley , Fractal Concepts in Surface Growth, (Cambridge University Press, New York, 1995).

[11] S.V.Ghaisas, cond-mat/0509684.

[12] M.Siegert, Phys. Rev. E 53, 3209 (1996).

[13] B.A. Mello, A.S. Chaves and F.A. Oliveira, Phys. Rev. E 63, 041113 (2001).

[14] S.V.Ghaisas, cond-mat/0307411. 
Figure 1: Plot of distribution of $\Delta h(t)_{l o c a l}$ for the $(1+1)$ dimensional model discribed in the text on semi $\log$ scale. The distribution is for $t=5000 \mathrm{MLs}$, with $\sigma=1.7$. The distribution is obtained by collecting the data over 3000 runs.

Figure 2: Plot of $P_{0}$ in $\%$ as a function of parameter $\sigma$ for the model in $(1+1)$ dimensions.

Figure 3: Plot of $G(x, t)$ Vs. $x$ on $\log -\log$ scale. The substrate size $\mathrm{L}=80000$, and the number of monolayers grown are $5 \times 10^{5}$.

Figure 4: Plot of $W_{\text {sat }}$ Vs. $L$ on $\log$-log scale for the model in $(1+1)$ dimensions.

Figure 5: Plot of $S(k, t)$ Vs. $k$ on log-log scale for the model in $(1+1)$ dimensions. The points are averaged over the substrate lengths of $L=800$, $900,1000,1100,1200,1300,1350 . k$ varies from $\pi / 100$ to $\pi$.

Figure 6: Plot of $W_{\text {sat }}$ Vs. $L$ on log-log scale for the model in $(2+1)$ dimensions (filled squares) and for the model in $(3+1)$ dimensions (filled circles).

Figure 7: Plot of $G(x, t)$ Vs. $x$ on $\log$-log scale for the model in $(2+1)$ dimensions. The substrate size is $L=800$, and the number of monolayers grown are 20000. 


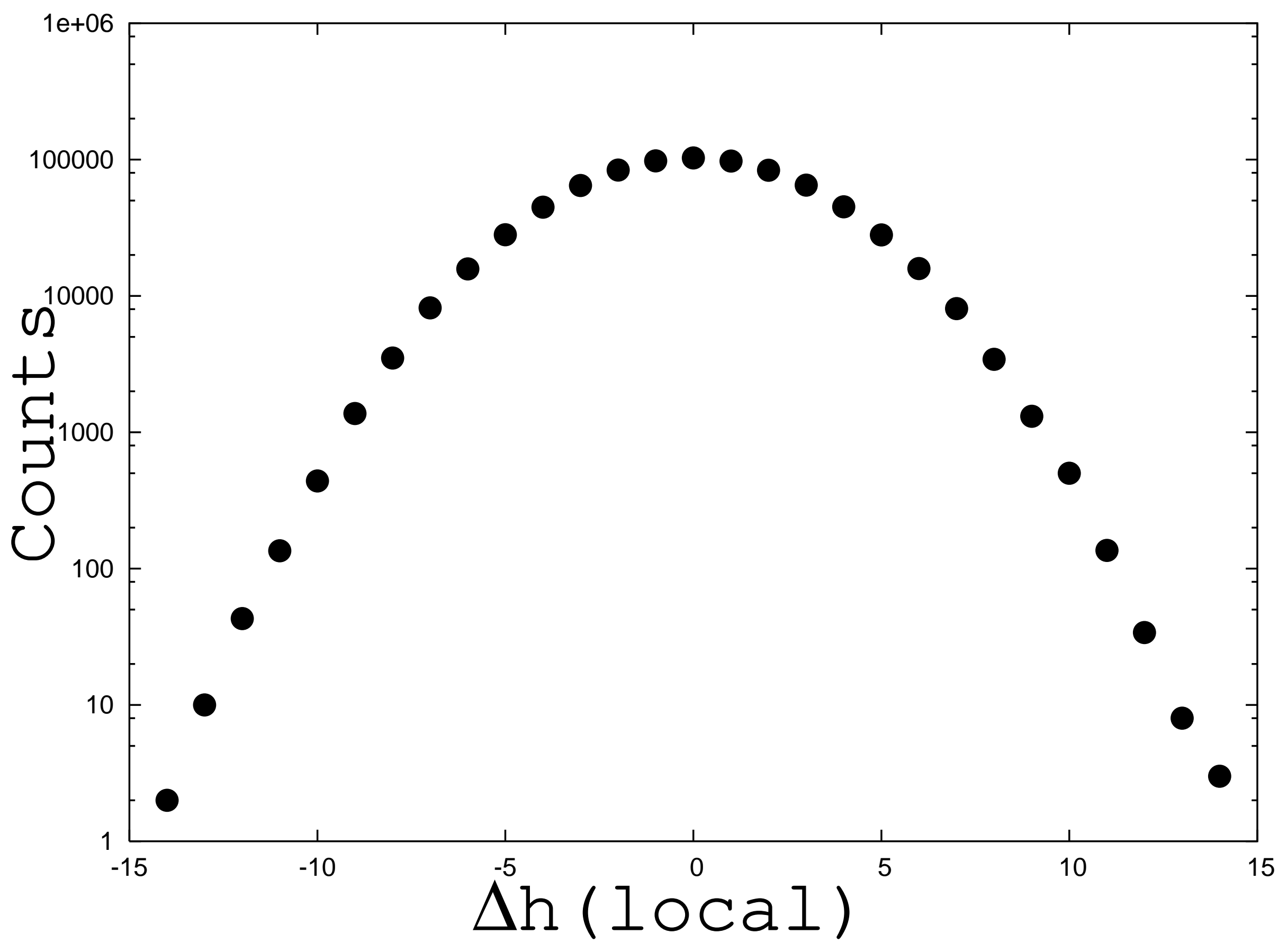




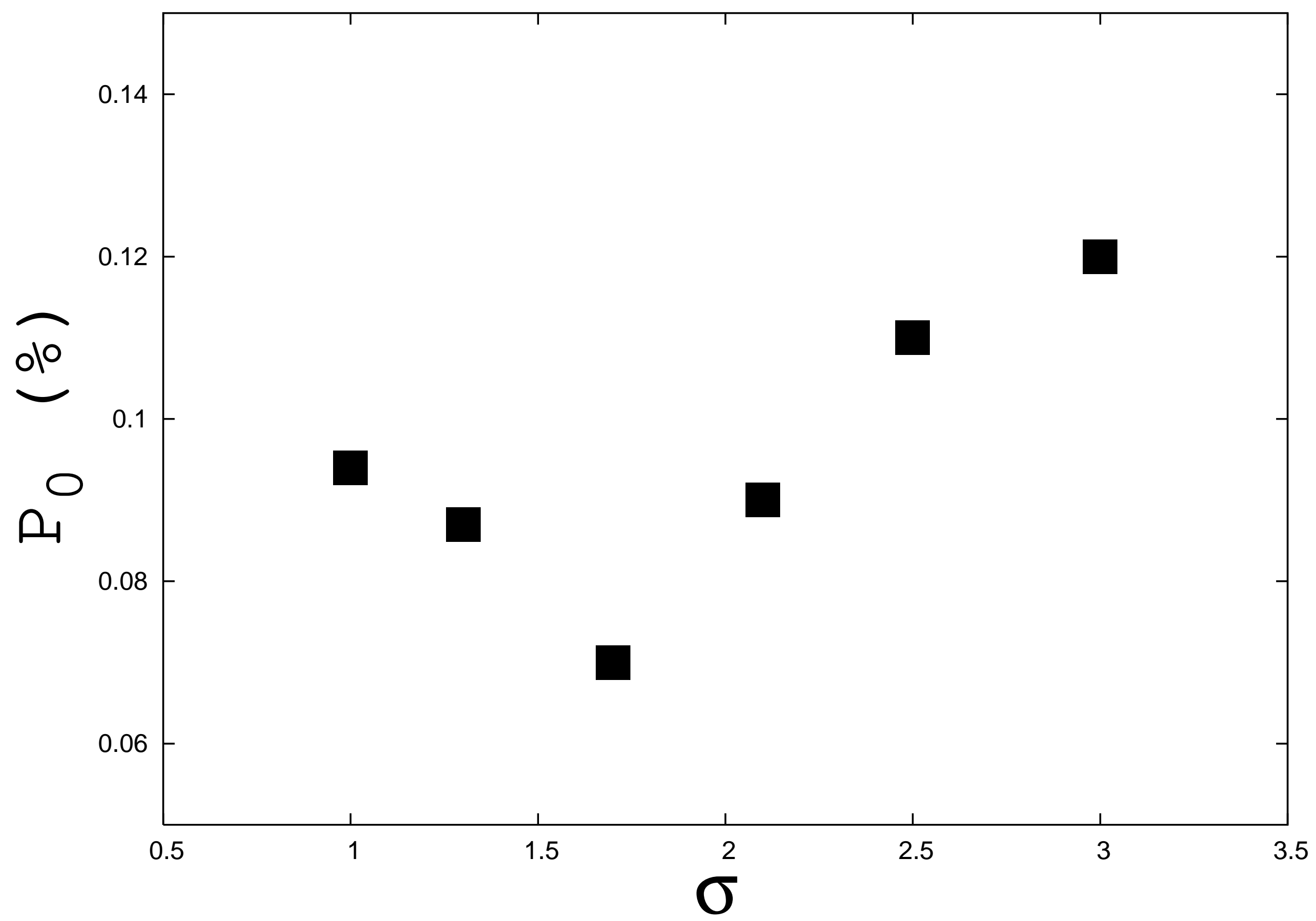




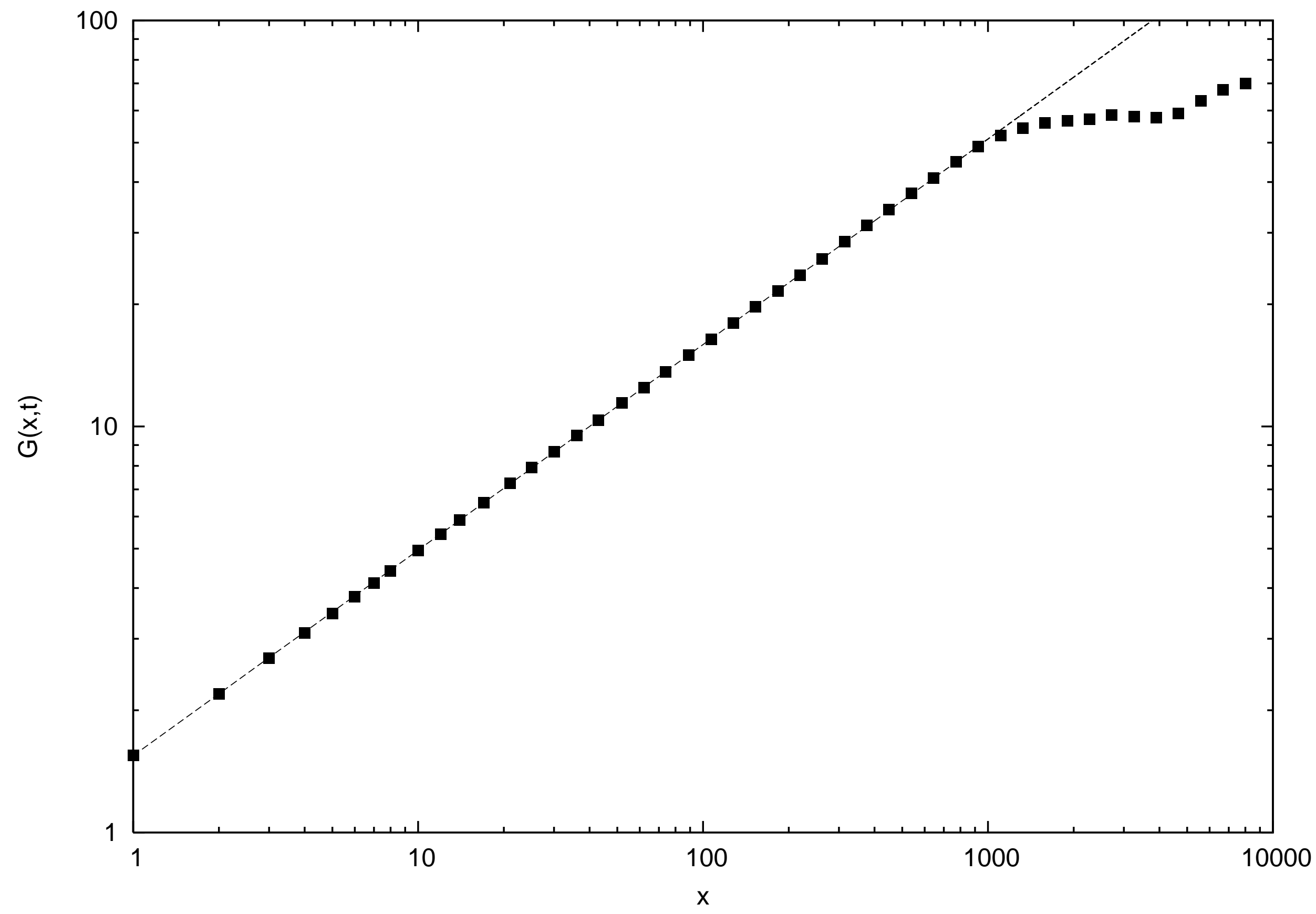




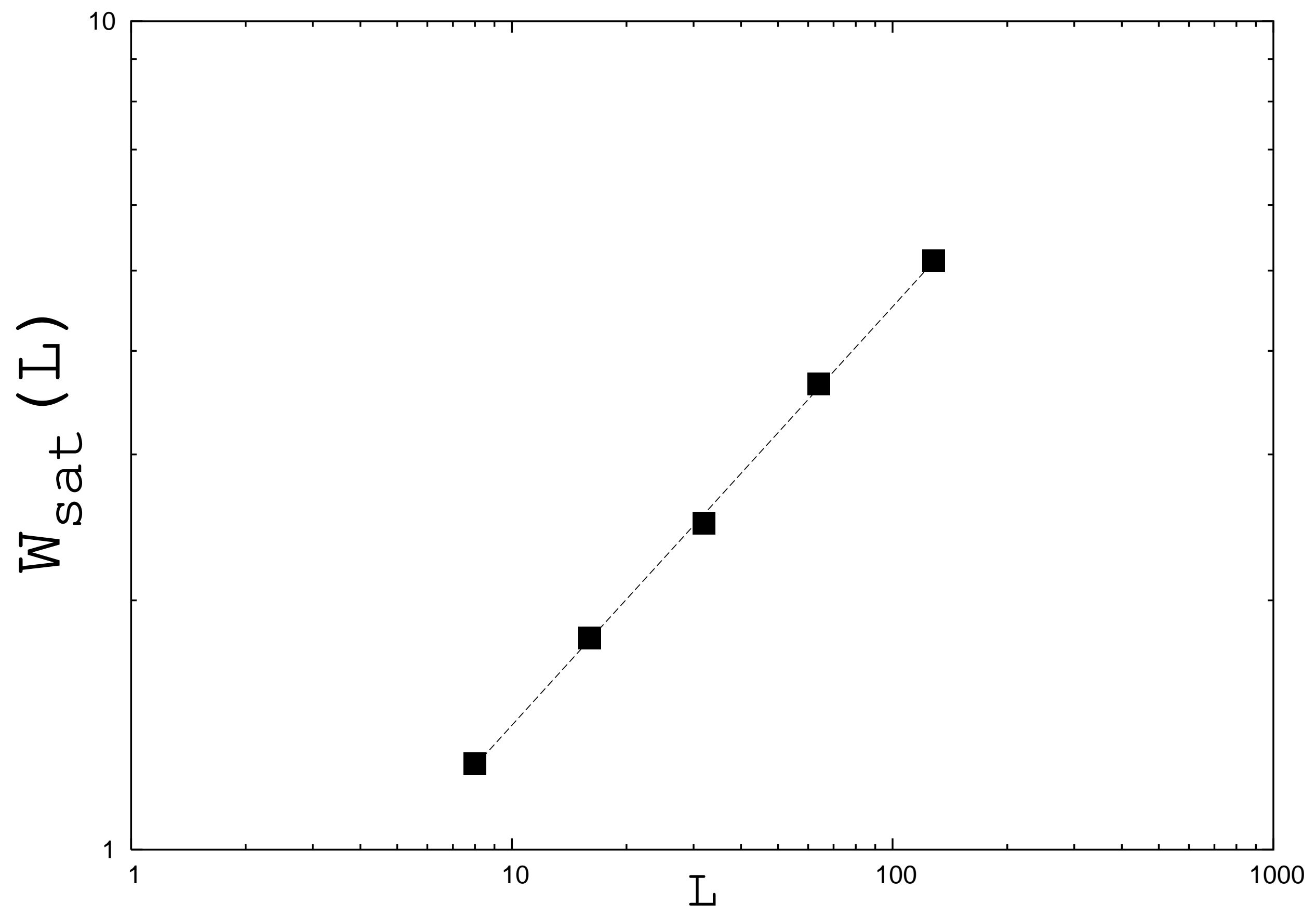




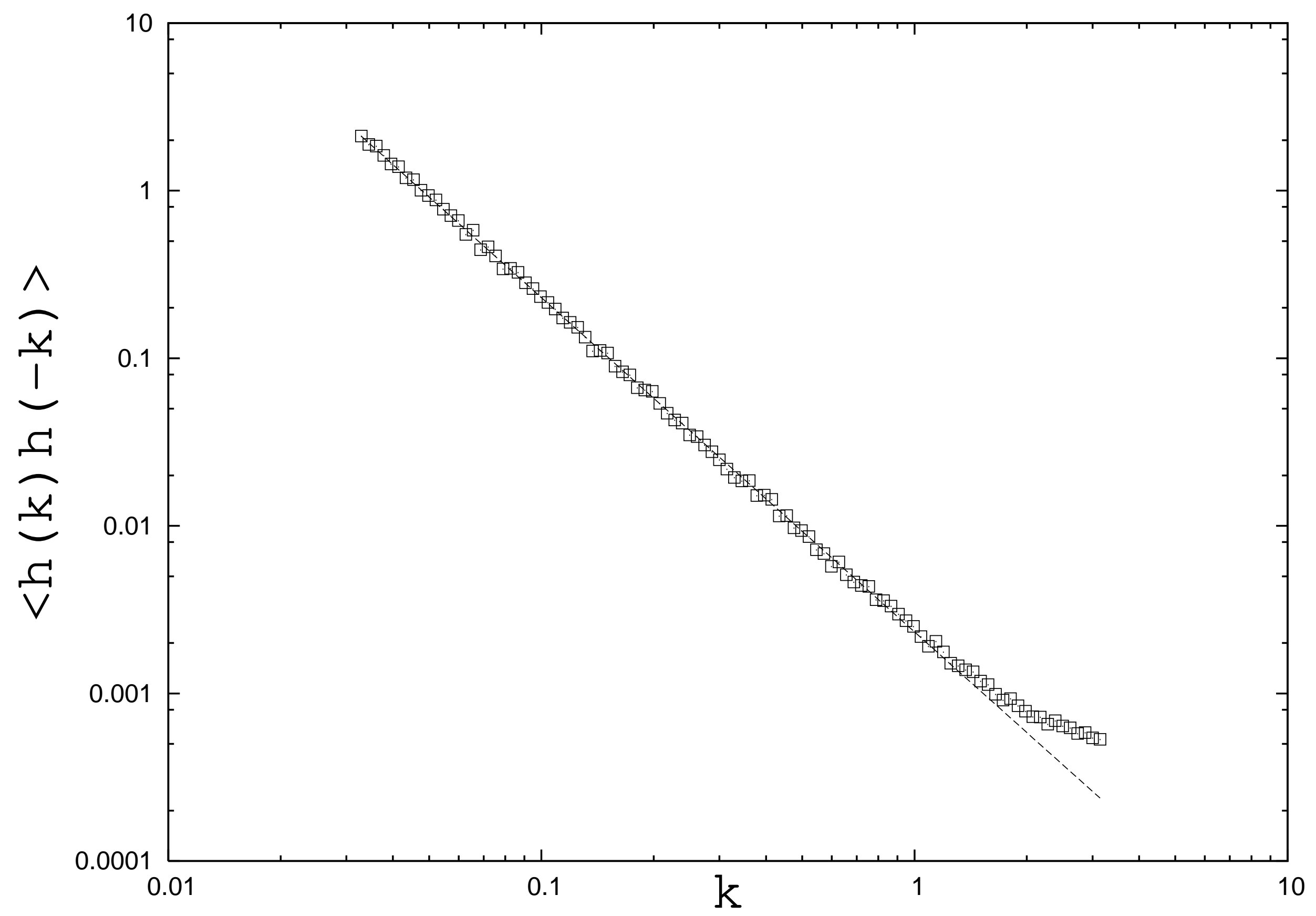




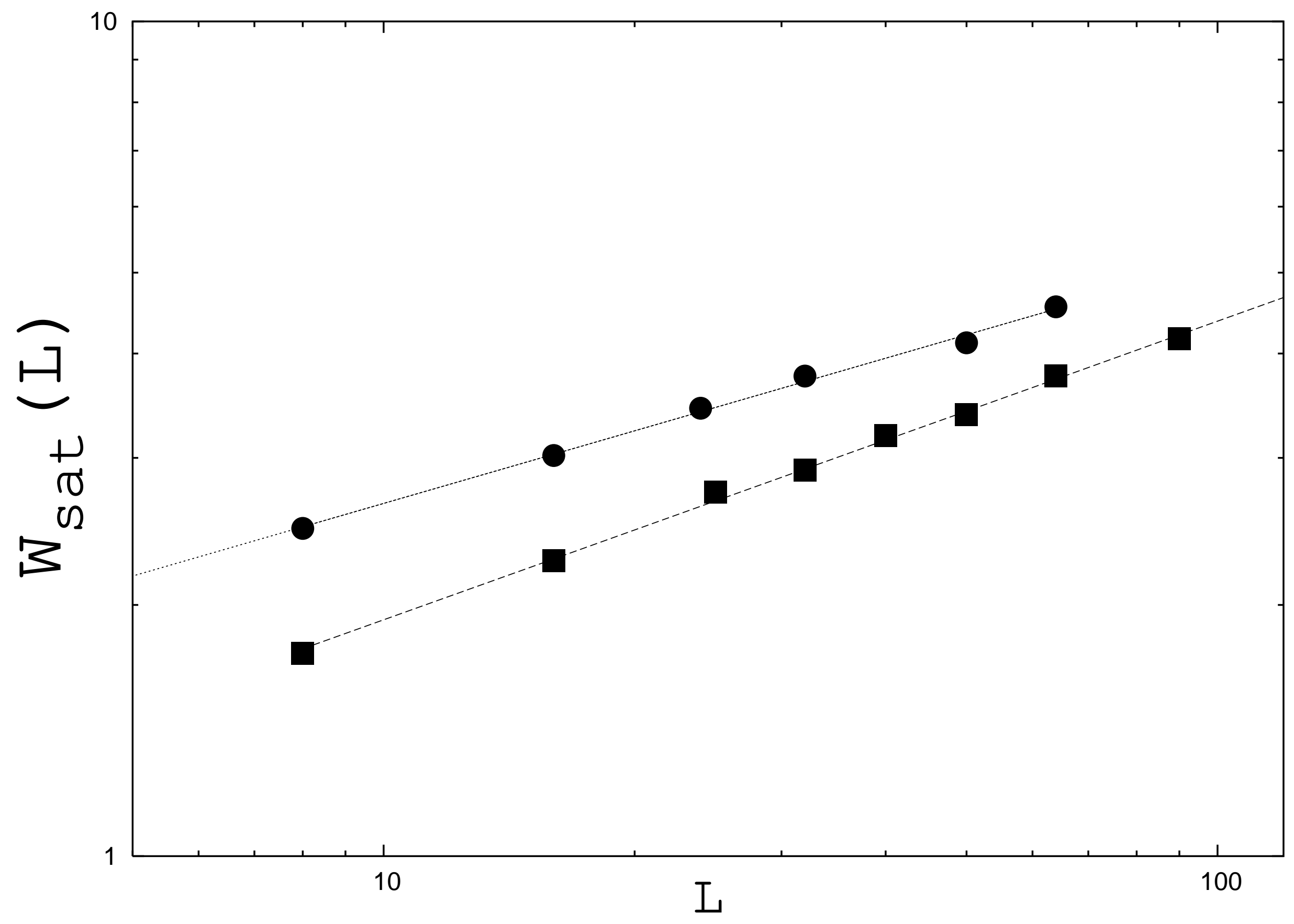




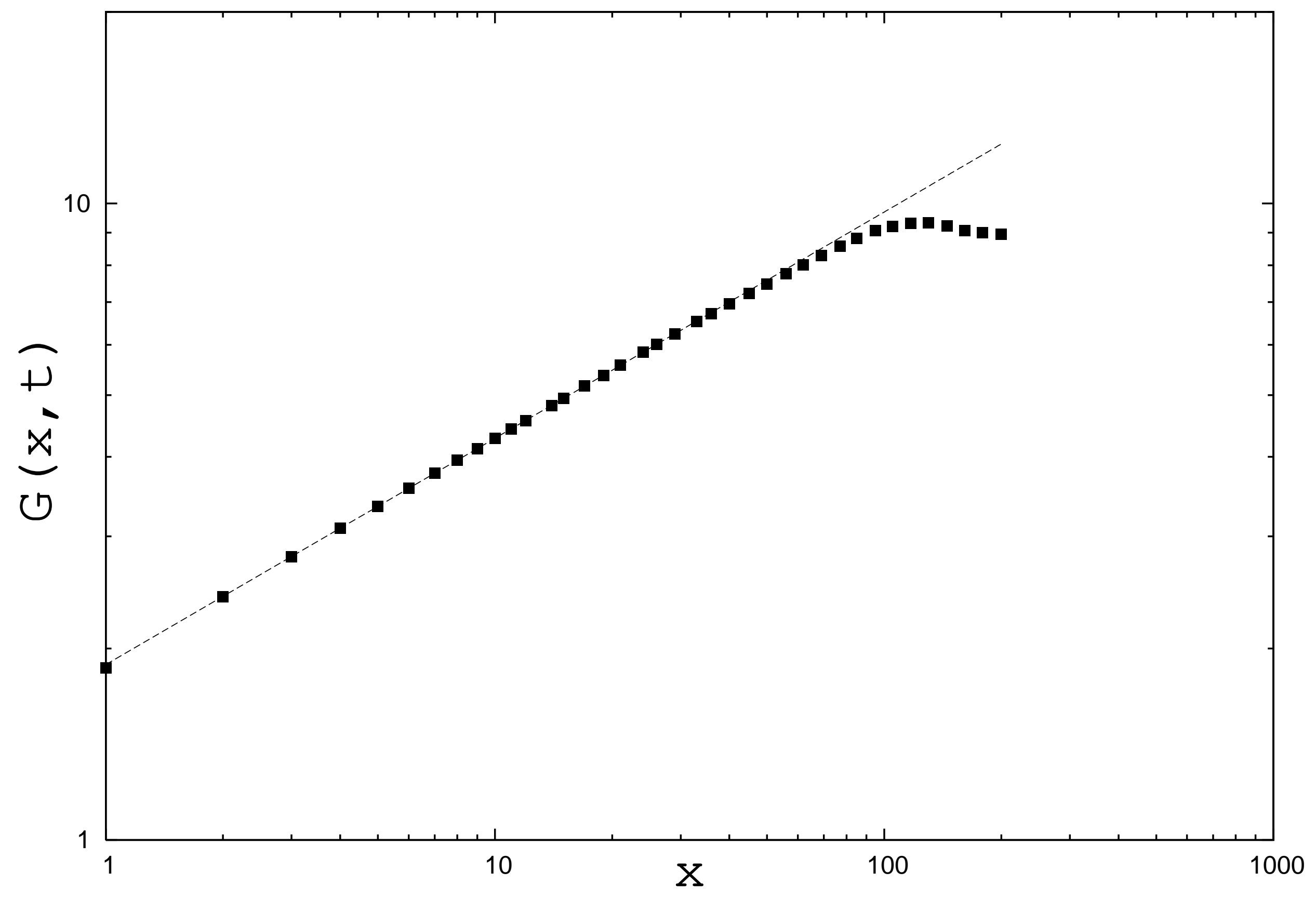

